\title{
Asking about dizziness when turning in bed predicts examination findings for benign paroxysmal positional vertigo
}

\author{
Ellen Lindell ${ }^{\mathrm{a}, *}$, Caterina Finizia ${ }^{\mathrm{a}}$, Mia Johansson $^{\mathrm{b}}$, Therese Karlsson ${ }^{\mathrm{a}}$, Jerker Nilson ${ }^{\mathrm{c}}$ \\ and Måns Magnusson ${ }^{\mathrm{d}}$ \\ ${ }^{a}$ Department of Otorhinolaryngology, Head and Neck Surgery, Institute of Clinical Sciences, \\ Sahlgrenska Academy at the University of Gothenburg, Sahlgrenska University Hospital, \\ Gothenburg, Sweden \\ ${ }^{\mathrm{b}}$ Department of Oncology, Institute of Clinical Sciences, Sahlgrenska Academy at the University \\ of Gothenburg, Sahlgrenska University Hospital, Gothenburg, Sweden \\ ${ }^{\mathrm{c}}$ Södra Älvsborgs Hospital, Borås, Sweden \\ ${ }^{\mathrm{d}}$ Department of Otorhinolaryngology Head and Neck Surgery, Clinical Sciences, Lund, Sweden
}

Received 2 February 2018

Accepted 18 July 2018

\begin{abstract}
.
BACKGROUND: Benign paroxysmal positional vertigo (BPPV) is the single most common cause of vestibular vertigo and is characterised by short episodes of rotational vertigo precipitated by changes in head positions like lying down or turning in bed.

OBJECTIVE: This study aims to assess useful questions when suspecting benign paroxysmal positional vertigo (BPPV) caused dizziness as well as identifying if a single question can be useful in identify or distinguish patients with BPPV from other dizziness aetiology.

METHOD: A total of 149 patients admitted due to dizziness were included. Patients answered a questionnaire and were investigated for BPPV with diagnostic manoeuvres.

RESULT: Two of the 15 questions were of diagnostic importance. Dizziness when laying down or turning in bed, increased likelihood of BPPV by an odds ratio (95\% confidence interval) of 60 (7.47-481.70). Continuous dizziness duration as opposed to lasting seconds decreased likelihood of BPPV with an odds ratio of $0.06(0.01-0.27)$.

CONCLUSION: Vertiginous attacks by turning or laying down in bed together with dizziness $<1$ minute, are important questions and strongly related to BPPV. Such questions are important when taking a medical history and may help to early identify BPPV, also for non-medical staff, as well as reduce the need of further investigations.
\end{abstract}

Keyword: BPPV, vertigo, diagnosis, dizziness, prevalence

\section{Abbreviations}

BPPV Benign Paroxysmal Positional Vertigo p-BPPV Posterior canal benign paroxysmal positional vertigo

\footnotetext{
${ }^{*}$ Corresponding author: Ellen Lindell, Department of Otolaryngology, Head and Neck Surgery, Sahlgrenska Universitetssjukhuset, Gröna Stråket 5, 41345 Göteborg, Sweden. Tel.: +46734 200 628; Fax: +46 336161638; E-mail: ellen.lindell@vg region.se.
}

h-BPPV Horisontal canal benign paroxysmal positional vertigo

DHI Dizziness Handicap Inventory

CI Confidence intervals

ENT Ear Nose Throat

ROC Receiver operating curve

\section{Introduction}

Dizziness, vertigo and imbalance are common complaints in both emergency and non-acute 
medicine and account for 1-2\% of consultations among outpatients in general practice $[8,12]$. Benign paroxysmal positional vertigo (BPPV) is one of the most common causes of peripheral vestibular vertigo $[2,11]$.

BPPV is characterised by short episodes of rotational vertigo precipitated by changes in head positions. The intense vertigo normally subsides if the head is held static. Sleep and time in bed appear to be of importance since many patients experience their first episode of vertigo in the morning.

The epidemiology of BPPV is not fully known and demographic analyses are few. The incidence of BPPV varies in studies from 6-17 per 100 000/year $[20,21]$ to a prevalence of between 1.4-2.4 [28, 31], however unrecognised BPPV among elderly has been noted as high as $9-11 \%$. [18, 23]. Right ear BPPV is more common than the left $[18,19,27,32]$, with more women affected than men (1.5:1) $[20,21,31,33]$ and the incidence increases with age $[28,31]$. Posterior canal BPPV (p-BPPV) is most common, followed by horizontal canal BPPV (h-BPPV) [15]. The recurrence rate of BPPV is relatively high, 16-27\% with most cases reported within the first months of the initial event $[1,3,24]$.

The diagnostic criteria for BPPV are vertigo combined with nystagmus provoked by Dix-Hallpike or supine roll test, latency before onset of nystagmus and fatigability $[6,11,30]$. The gold standard for diagnosis of BPPV is established using the Dix-Hallpike manoeuvre (DH) for p-BPPV and the supine roll test (LR) for detecting h-BPPV [6, 11]. The sensitivity for Dix-Hallpike för BPPV in the posterior canal is estimated to be around $80 \%$ [7] and others have reported positive findings for BPPV after repetition of the DixHallpike manoeuvre even if tested negative initially $[9,29]$.

When detected, BPPV is usually easy to treat using the Epley or Semont manoeuvre leading to accelerated recovery and thereby less suffering [15]. Without treatment, effects for the patient can be significant with one study finding that $86 \%$ of affected individuals had to seek medical consult, apply for sick leave or have their daily activities interrupted [31].

Few validated questionnaires for diagnoses in the dizzy patient exist. The most used questionnaire, Dizziness Handicap Inventory (DHI), has been found useful as high DHI-scores may predict BPPV [26]. However, others find it less useful as self-perceived handicap and a high DHI score do not necessarily correlate with disturbed balance as measured clinically [13]. This is well in line with the purpose of the instrument, as it is designed to recognize the impact of dizziness and not to be a diagnostic tool. This demonstrates the difficulties when developing a standardized questionnaire addressing the subjective and diverse spectra of complaints found in the dizzy patient. Asking for duration of dizziness and symptoms provoked by changes of head position have been shown useful questions in detecting probable BPPV $[22,31]$.

As BPPV is the most commonly diagnosed cause of vestibular dizziness [11], and one that most often can be treated, any easy and early applied discriminatory action with substantial diagnostic yield, will be of paramount impact in everyday medical practice. Even more so if possible to be taught to auxiliary staff. Hence, this study aims to assess useful questions when suspecting BPPV-caused dizziness as well as identifying if a single question can distinguish patients with BPPV from other dizziness aetiology.

\section{Method}

\subsection{Study population}

Patients referred to the Ear, Nose and Throat clinic at Södra Älvsborgs Hospital, Sweden, a secondary referral centre, between the years 2013 and 2014, were eligible for the study. Inclusion criteria were seeking medical care due to dizziness or imbalance and being over 18 years of age with absent neurological signs or cervical instability. Patients included were either referred non-acutely from their general practitioner/by own referral $(72 \%)$ or acutely from the emergency room (28\%). 160 patients were invited and 149 patients accepted to participate in the survey. Waiting time for patients admitted from their general practitioner was weeks to a maximum of three months whereas waiting time for patients admitted acutely was hours to a maximum of three days. The patients were examined by a doctor $(67 \%)$ or by a vestibular assistant (33\%). Patients were seen directly by the vestibular assistant when BPPV was strongly suspected from the referral notes. All patients examined by the vestibular assistant or research leader were consecutively invited to participate in the study. All patients included in the study were with very few exceptions investigated by the same senior resident or a single vestibular assistant. 
Table 1

Study-specific questionnaire

\begin{tabular}{lc}
\hline Question & Response options \\
\hline Q1. Do you get dizzy when laying down or turning over in bed? & Yes/No \\
Q2. If so, which side is worse? & Left/Right/Both/Neither \\
Q3. Do you get dizzy when sitting up? & Yes/No \\
Q4. Do you get dizzy when standing up? & Yes/No \\
Q5. Preferred head-laying side during sleeping? & Left/Right/Both/Neither \\
Q6. For how long does the dizziness last? & Yes/No \\
Q7. Can you get dizzy without positional change? & Yes/No \\
Q8. Are you unsteady by gait? & Yes/No \\
Q9. Have you experienced previous head trauma? & Yes/No \\
Q10. Recent prolonged bed rest? & Yes/No \\
Q11. Previous ear disease? & Yes/No \\
Q12. Previous similar symptoms? & Yes/No \\
Q13. Do you have neck problems? & Yes/No \\
Q14. Do you have double vision? & Yes/No \\
Q15. New hearing impairment? &
\end{tabular}

\subsection{Study design}

In this cross-sectional study, all 149 participating patients were during their visit in the ENT-department first asked a series of questions (Table 1) from a study-specific questionnaire. Patients were interviewed and the investigator filled in the questionnaire. Directly thereafter the participants underwent a physical examination performed by the same investigator Examination included the Head-Shake Test, the Head Impulse Test, positioning tests (Dix-Hallpike manoeuvre and supine roll test) as well as assessing the presence of spontaneous or gaze evoked nystagmus, with direction and duration being observed with and without Video Frenzel (Synapsys Video Nystamoscopy Goggle VNS 3X). The diagnosis of BPPV was made if Dix-Hallpike or supine roll test triggered vertigo and canal specific nystagmus was seen.

\subsection{Study-specific questionnaire}

Since few validated questionnaire regarding dizziness and BPPV exists with conflicting reported usefulness [13], a study-specific questionnaire was used. This questionnaire consisted of 15 questions focusing on the presence of dizziness, potential triggers and associated symptoms (Table 1). Questions were chosen and formulated based on literature, clinical expertise and comments from patients and the examiner (physician or vestibular assistant) asked the questions. If the patient could not give affirmative answers, they were asked to choose the option they considered most appropriate, alternatively, the question was left unanswered.

\subsection{Statistical analysis}

Mean, median and $95 \%$ confidence intervals (CI) of the mean were used for descriptive purposes. Mean and CI were used for continuous variables whilst number and percent were used for categorical variables. For comparison between two groups Fisher's exact test was used for dichotomous variables and Mann Whitney U-test for continuous variables. In order to find variables indicative of having a BPPVdiagnosis, uni- and multivariable logistic regression analyses were performed. The result was presented as an odds ratio with $95 \% \mathrm{CI}$, corresponding $p$-value and area under Receiver operating curve, (ROC-curve) with $95 \%$ CI. All significance tests were two-sided and conducted at 5\% significance level. The SPSS version 20.0 for Mac and SAS 9.3 was used for analyses.

\subsection{Ethical considerations}

The study was conducted according to the Declaration of Helsinki and was approved by the Regional Ethic Review Board in Gothenburg, Sweden. Written informed consent for participation in the study was obtained from all participants

\section{Results}

A total of 149 patients were included in the study, of which $36 \%(n=53)$ were male and $64 \%(n=96)$ 
were female. Fifty-nine patients were diagnosed with BPPV, with $27 \%$ being male and $73 \%$ female. The remaining 90 had other dizziness aetiologies and a gender distribution of $41 \%$ and $59 \%$ respectively (Table 2). There was no statistically significant difference in terms of gender and age in the non-BPPV and the BPPV-group.

Table 2

Sociodemographic data of the study population

\begin{tabular}{lcccc}
\hline & $\begin{array}{c}\text { Total } \\
n=149 \\
\mathrm{n}(\%)\end{array}$ & $\begin{array}{c}\text { No BPPV } \\
n=90 \\
\mathrm{n}(\%)\end{array}$ & $\begin{array}{c}\text { BPPV } \\
n=59\end{array}$ & $p$-value \\
& & & & \\
\hline Gender & & & \\
$\quad$ Male & $53(36 \%)$ & $37(41 \%)$ & $16(27 \%)$ & \\
$\quad$ Female & $96(64 \%)$ & $53(59 \%)$ & $43(73 \%)$ & 0.11 \\
Age years & & & & \\
$\quad$ Mean (median) & $59.5(61.0)$ & $58.5(57.0)$ & $61.0(64.0)$ & 0.37 \\
\hline
\end{tabular}

\subsection{Study-specific questionnaire items and prediction of BPPV diagnosis}

Of the items in the study-specific questionnaire, univariable analysis revealed three items demonstrating a significant relationship with a positive BPPV diagnosis, namely Q1 - experiencing dizziness when laying down or turning in bed, Q3 - experiencing dizziness when sitting up and Q6 - the duration of dizziness (Fig. 1). The relationships are quantified in Table 3. However, only Q1 and Q6 remained significant on multivariate analysis. The remaining questions showed no statistically significant odds ratios indicating an increased risk of BPPV-diagnosis as well as having low areas under the ROC-curve (0.50-0.58).

Of the 94 patients who replied "yes" to being dizzy when laying down in bed or turning over in bed

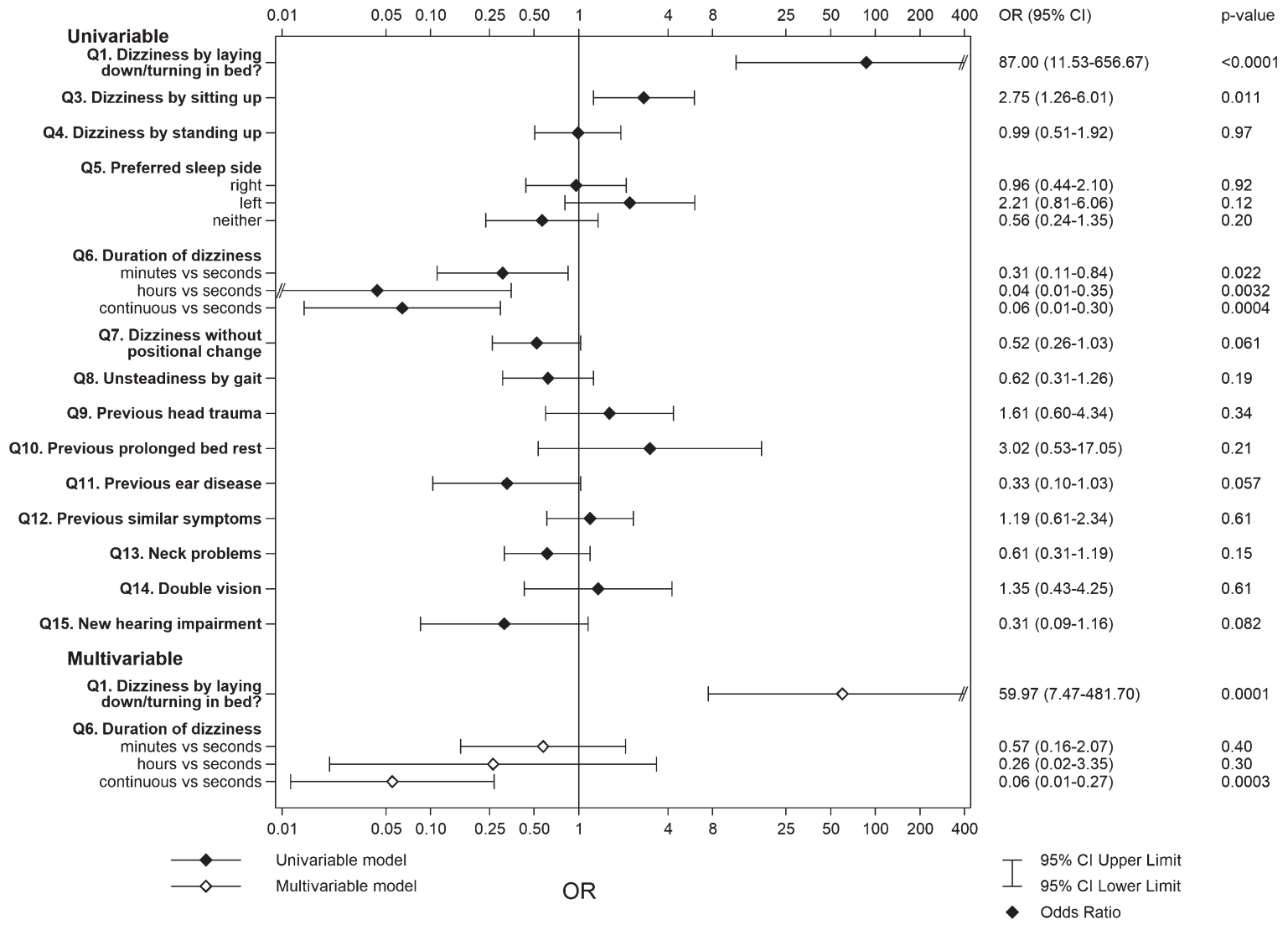

Fig. 1. Forrest plot describing the odds ratio between the study-specific questionnaire items and a positive BPPV diagnosis. OR = odds ratio, $\mathrm{CI}=$ confidence interval. OR represents the odds that an outcome will occur given the particular exposure, compared to the odds of the outcome occurring in the absence of the exposure and is measured as odds of outcome per unit increase in the value of the exposure. $\mathrm{OR}=1$ : exposure does not affect odds of outcome, OR $>1$ : exposure is associated with higher chance of outcome, OR $<1$ : exposure is associated with lower chance of outcome. 
Table 3

Univariable and multivariable analysis of study-specific questionnaire items and diagnosis of BPPV

\begin{tabular}{|c|c|c|c|c|c|c|c|c|}
\hline & \multirow[b]{2}{*}{ Missing } & \multicolumn{4}{|c|}{ Univariable analysis $(n=149)$} & \multicolumn{3}{|c|}{ Multivariable analysis } \\
\hline & & Answer & $\begin{array}{c}\mathrm{n}(\%) \text { of } \\
\text { event }\end{array}$ & $\begin{array}{c}\text { OR } \\
(95 \% \mathrm{CI}) \\
\text { BPPV } \\
\end{array}$ & $\overline{p \text {-value }}$ & $\begin{array}{l}\text { Area under } \\
\text { ROC-curve }\end{array}$ & $\begin{array}{c}\text { OR } \\
(95 \% \mathrm{CI}) \\
\text { BPPV } \\
\end{array}$ & $\overline{p \text {-value }}$ \\
\hline \multirow{2}{*}{$\begin{array}{l}\text { Q1. Dizziness } \\
\text { when laying down/ } \\
\text { turning in bed }\end{array}$} & \multirow[t]{2}{*}{0} & No & $1(1.8 \%)$ & & & & & \\
\hline & & Yes & $58(61.7 \%)$ & $\begin{array}{c}87.00 \\
(11.53-656.67) \\
\end{array}$ & $<0.0001$ & 0.79 & $\begin{array}{c}59.97 \\
(7.47-481.7) \\
\end{array}$ & 0.0001 \\
\hline \multirow{2}{*}{$\begin{array}{l}\text { Q3. Dizziness } \\
\text { when sitting up }\end{array}$} & \multirow[t]{2}{*}{2} & No & $11(24.4 \%)$ & & & & & \\
\hline & & Yes & $48(47.1 \%)$ & $\begin{array}{c}2.75 \\
(1.26-6.01) \\
\end{array}$ & 0.011 & 0.60 & & \\
\hline \multirow{2}{*}{$\begin{array}{l}\text { Q4. Dizziness } \\
\text { when standing up }\end{array}$} & \multirow[t]{2}{*}{2} & No & $25(40.3 \%)$ & & & & & \\
\hline & & Yes & $34(40.0 \%)$ & $\begin{array}{c}0.99 \\
(0.51-1.92)\end{array}$ & 0.97 & 0.50 & & \\
\hline \multirow{4}{*}{$\begin{array}{l}\text { Q6. Duration of } \\
\text { dizziness }\end{array}$} & \multirow[t]{4}{*}{13} & Seconds & $49(62 \%)$ & 1.0 & & & 1.0 & \\
\hline & & Minutes & $7(33.3 \%)$ & $\begin{array}{c}0.31 \\
(0.11-0.84) \\
\end{array}$ & 0.022 & & $\begin{array}{c}0.57 \\
(0.16-2.07) \\
\end{array}$ & 0.40 \\
\hline & & Hours & $1(6.7 \%)$ & $\begin{array}{c}0.04 \\
(0.01-0.35) \\
\end{array}$ & 0.0032 & & $\begin{array}{c}0.26 \\
(0.02-3.35) \\
\end{array}$ & 0.30 \\
\hline & & Continuous & $2(9.5 \%)$ & $\begin{array}{c}0.06 \\
(0.01-0.30) \\
\end{array}$ & 0.0004 & 0.74 & $\begin{array}{c}0.06 \\
(0.01-0.27) \\
\end{array}$ & 0.0003 \\
\hline \multirow{2}{*}{$\begin{array}{l}\text { Q7. Dizziness } \\
\text { without positional } \\
\text { change }\end{array}$} & \multirow[t]{2}{*}{0} & No & $40(46 \%)$ & & & & & \\
\hline & & Yes & $19(30.6 \%)$ & $\begin{array}{c}0.52 \\
(0.26-1.03)\end{array}$ & 0.06 & 0.58 & & \\
\hline
\end{tabular}

Abbreviations: $\mathrm{BPPV}=$ benign paroxysmal positional vertigo, $\mathrm{CI}=$ confidence interval, $\mathrm{OR}=$ odds ratio, $\mathrm{ROC}=$ receiver operating characteristic.

(Q1), there was no statistically significant difference in terms of which side the head was turned when the dizziness occurred $(p=0.17)$. Of these 94 patients, 58 were diagnosed with BPPV yielding a point prevalence of $61.7 \%$ compared to the one patient with a BPPV-diagnosis amongst patients $(n=55)$ answering "no" to question, resulting in a corresponding point prevalence of $1.8 \%$ (Table 3 ). The odds ratio of having BPPV was 87 times higher $(p<0.0001)$ given an affirmative answer to this question. On multivariate analysis this figure was 60 times higher $(p=0.0001)$. The sensitivity for the question of dizziness by turning or lying down was $98 \%$ and the specificity $60 \%$ with a negative predictive value for Q1 of 0,98 and positive predictive value of 0.62 . Of those replying that dizziness lasted only seconds, $62 \%(n=49)$ were diagnosed with BPPV. Corresponding figures for dizziness lasting minutes, hours or being continuous (Q6) were $33.3 \%(n=7), 6.7 \%$ $(n=1)$ and $9.5 \%(n=2)$ (Table 3$)$. The sensitivity and specificity for Q6 is shown diagrammatically in Fig. 2, where the true positive rate (sensitivity) is plotted graphically against the false negative rate (1specificity) in a receiver operating curve. Reporting that dizziness lasted only seconds as opposed to being continuous was a significant predictor of BPPV in the multivariate analysis $(p<0.001)$. The probability of having BPPV if reporting dizziness when laying down or turning in bed and replying that dizziness lasts only seconds resulted in a probability of 0,73 $(0,62-0,83)$. Of the patients reporting dizziness when laying down or turning over in bed but where no nystagmus was seen and therefore no BPPV diagnosis was set $(n=36), 13$ had a history with dizziness suspicious of BPPV, three suffered from vestibular neuritis, one from Meniere's disease (on-going attack), three had a central cause of vertigo (stroke or transient ischemic attack), two had acute audiovestibular loss, three had probable cervical vertigo and 11 unknown cause of dizziness. During testing, 12 of the 149 patients had positive horizontal Head Shake nystagmus. Of these 12, eight had BPPV (four pBPPV, four hBPPV), one Ménières disease, two auditory loss with accompanying dizziness and one patient suffered from dizziness after head trauma. One patient with BPPV had spontaneous nystagmus. No patient with BPPV had a positive Head Impuls test.

\section{Discussion}

Dizziness when turning in bed is highly correlated to BPPV. The response to a question of turning in bed 


\section{Sensitivity and 1-specificity for question 6: BPPV and duration of dizziness \\ ROC Curve for Model}

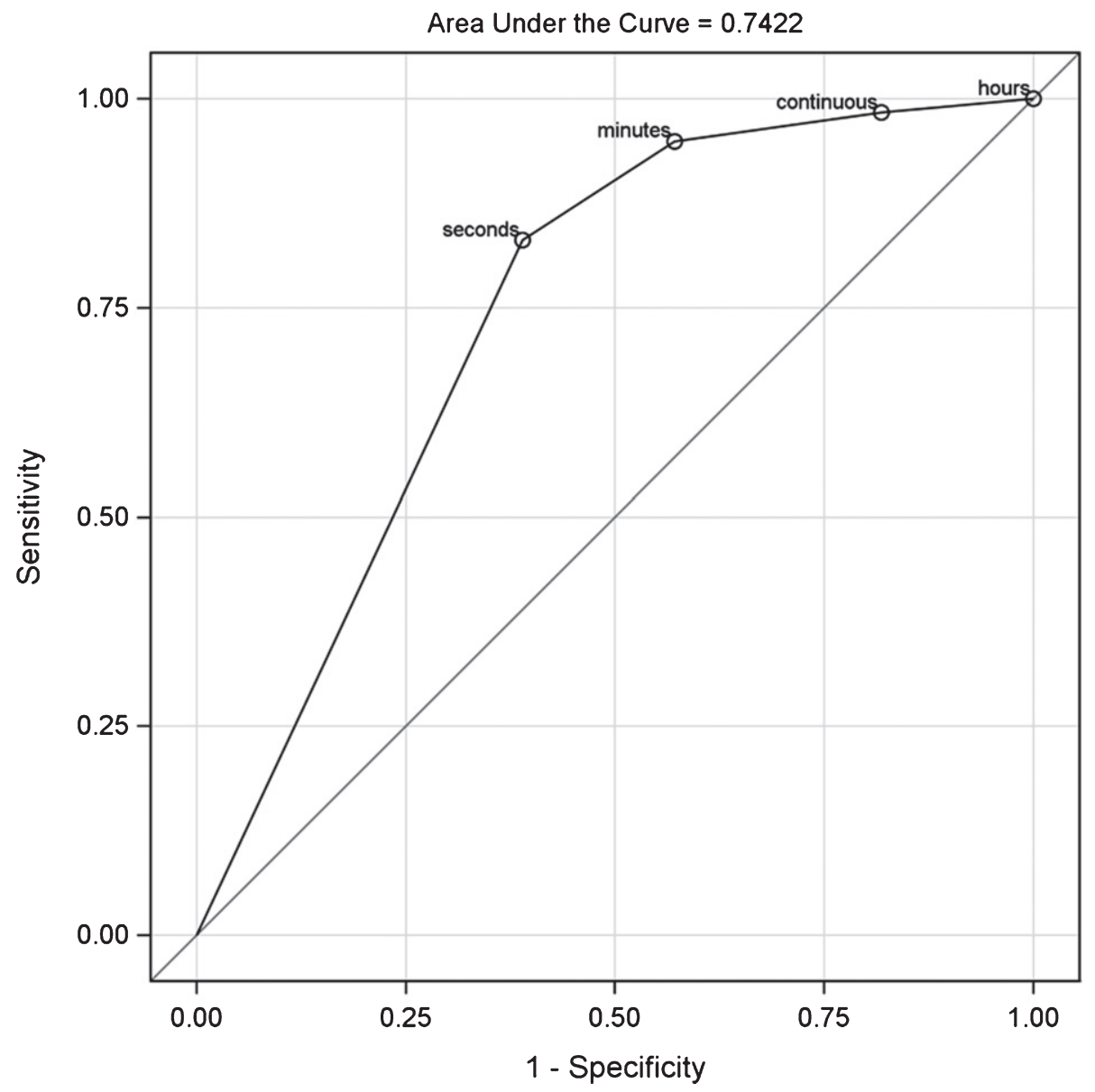

Fig. 2. ROC-curve describing the sensitivity and 1-specificity for question 6: BPPV and duration of dizziness. ROC $=$ Receiver operating curve.

as provocative for dizziness identified patients with a clinical diagnosis of BPPV at $98 \%$ sensitivity and $60 \%$ specificity. A negative response to this question was $98 \%$ accurate in ruling out BPPV in a sample with a $39 \%$ prevalence. Reporting episodes of vertigo lasting seconds as opposed to longer duration found BPPV with a sensitivity of $83 \%$. To our knowledge, there have been few studies addressing the possibility of screening for BPPV with only a few questions. The possibility of early identification of possible BPPV or its exclusion, may enhance productivity, speed up treatment and reduce health risks with missed diagnosis in patient with acute or semi acute onset of dizziness [5, 17]. The results confirmed that two questions were particularly useful for this purpose. Experiencing vertigo when laying down or turning over in bed and combined with a vertigo-episode lasting seconds as opposed to being continuous, are strong predictors of BPPV. This is in line with findings in the literature where von Brevern et al. [31] reported vertiginous attacks in bed in all their patients with BPPV. Similarly, Noda et al found that the two most important questions regarding BPPV features were onset of dizziness when turning over in bed and dizziness - of any kind - lasting $\leq 15$ seconds [22]. Noda et al. made their findings in a general medicine department from unselected outpatients with complaints of dizziness in Japan. However, in contrast to Noda et al, this study is set in a secondary emergency hospital including both emergency and outpatient subjects. This study confirms the findings by Noda et al that dizziness in bed together with dizziness 
Table 4

Preferred sleeping side and affected semicircular canal in patients diagnosed with BPPV presented as number and percentage

\begin{tabular}{lccccc}
\hline & $\begin{array}{c}\text { Posterior right } \\
(n=31)\end{array}$ & $\begin{array}{c}\text { Posterior left } \\
(n=14)\end{array}$ & $\begin{array}{c}\text { Cupulolithiasis } \\
(n=6)\end{array}$ & $\begin{array}{c}\text { Lateral } \\
(n=8)\end{array}$ & $p$-value \\
\hline Preferred sleeping side & $\mathrm{n}(\%)$ & $\mathrm{n}(\%)$ & $\mathrm{n}(\%)$ & $\mathrm{n}(\%)$ & \\
$\quad$ Right & $12(46)$ & $6(46)$ & $1(20)$ & $7(88)$ & \\
Left & $6(23)$ & $5(39)$ & $3(60)$ & $0(0)$ & \\
Neither & $8(31)$ & $2(15)$ & $1(20)$ & $1(12)$ & 0.14 \\
\hline
\end{tabular}

$<1$ minute are useful questions also in the acute phase when diagnosing origin for dizziness.

Here, women presented with vertigo and imbalance more frequently than males, and a larger proportion of patients diagnosed with BPPV were women ( $73 \%$ vs $27 \%$ ), in line with previous reports $[8,28]$. Data also confirmed that the right posterior semicircular canal was the most frequently affected (Table 4).

Although the study population was not comprised of all patients presenting with dizziness, but only those referred to the ENT department, asking about dizziness when turning over or laying down in bed, and for short duration of dizziness still returned a high diagnostic yield, highlighting to the usefulness of such an approach when investigating dizzy patients. In this study, only patients with characteristic nystagmus were diagnosed with BPPV according to the diagnostic BPPV criteria formulated by the Committee for Classification of Vestibular Disorders of the Barany society [30]. Patients with suspected BPPV (dizziness triggered by turning in bed lasting less than a minute) but no nystagmus were offered a reexamination at the clinic if their symptoms remained after two weeks.

One limitation to this study might be that the study persons first were asked through the questionnaire and were then examined by the same investigator, hence risking some bias, since no blinding. However, Dix-Hallpike maneuver has been shown to have high levels of agreement between tests [4] and although there is a risk of false positive BPPV and false negative non-BPPV diagnoses, the DixHallpike test is well-established as an acceptable test to aid diagnosis of BPPV. Additionally, the width of the confidence interval for Q1 (Table 3, Fig. 1) is due to the patient inclusion selection, yielding a strong tendency toward those with a BPPV diagnosis, where nearly all patients had answered affirmatively on Q1 $(n=58)$ as opposed to negatively $(n=1)$. Since waiting time from referral to examination was weeks it is possible that spontaneous remission may have occurred.

\subsection{Clinical implications}

Vertigo triggered by turning over in bed together with vertigo lasting less than a minute are valuable amnestic indicators, which should be useful in an outpatient or general practitioner setting, where dizziness is a frequent problem with a multitude of aetiologies. The possibility of identifying probable BPPV with high accuracy is time-sparing as it aids management and appropriate onward referral. Since no other questions demonstrated significance, these could possibly be omitted when screening for BPPV.

Furthermore, asking those questions could be used by all care providers to find patients who could be easily be helped and hence, reduce risk of falls and health costs among especially the elderly. It could also serve as a detector when not to suspect BPPV as the cause of symptoms and thus, direct the attention to other causes of malady.

However, it should be pointed out that strong rotatory vertigo is somewhat less commonly reported in the elderly population $[10,25]$. One possible explanation being the decreased sensitivity of the vestibular organ and vestibular reflexes with age. While younger patients report a four times higher presence of 'true' vertigo (an illusion of movement) compared to elderly, the latter more often describe their symptoms as a giddiness or a tendency to fall [25]. Nevertheless, dizziness is a frequent complaint in the elderly and BPPV is common in this group. As BPPV is common among elderly $[14,16,18,28]$ the attending physician should be extra generous with investigating elderly complaining for dizziness or vertigo, especially those who report being dizzy when rolling over in bed, with the Dix-Hallpike maneuvers and supine roll test.

\section{Conclusion}

Vertiginous attacks when turning over in bed are strongly related to BPPV. Asking for vertigo by turning or laying down in bed together with vertigo $<1$ minute are rewarding questions when taking 
a medical history and may help to early identify BPPV as well as reduce the need of further investigations. Hence, medical treatment of the dizzy patient could be rationalized by methodically introducing this question early on in the medical assessment.

\section{Acknowledgments, competing interests and funding}

No competing interests exist. This study was supported by the Local Research and Development Council, Södra Älvsborg and the Ear, Nose and Throat Department at Södra Älvsborg Hospital as well as Swedbank Sjuhärad Foundation.

\section{References}

[1] S. Babac, D. Djeric, M. Petrovic-Lazic, N. Arsovic and A. Mikic, Why do treatment failure and recurrences of benign paroxysmal positional vertigo occur? Otol Neurotol 35 (2014), 1105-1110.

[2] N. Bhattacharyya, S.P. Gubbels, S.R. Schwartz, J.A. Edlow, H. El-Kashlan, T. Fife, J.M. Holmberg, K. Mahoney, D.B. Hollingsworth, R. Roberts, M.D. Seidman, R.W. Steiner, B.T. Do, C.C. Voelker, R.W. Waguespack and M.D. Corrigan, Clinical Practice Guideline: Benign Paroxysmal Positional Vertigo (Update), Otolaryngol Head Neck Surg 156 (2017), S1-s47.

[3] T. Brandt, D. Huppert, J. Hecht, C. Karch and M. Strupp, Benign paroxysmal positioning vertigo: A long-term follow-up (6-17 years) of 125 patients, Acta Otolaryngol 126 (2006), 160-163.

[4] A. Burston, S. Mossman and M. Weatherall, Are there diurnal variations in the results of the Dix-Hallpike manoeuvre? J Clin Neurosci 19 (2012), 415-417.

[5] C.E. Corrales and N. Bhattacharyya, Dizziness and death: An imbalance in mortality, Laryngoscope (2016).

[6] M.R. Dix and C.S. Hallpike, The pathology, symptomatology and diagnosis of certain common disorders of the vestibular system, Ann Otol Rhinol Laryngol 61 (1952), 987-1016.

[7] J. Dros, O.R. Maarsingh, H.E. van der Horst, P.J. Bindels, G. Ter Riet and H.C. van Weert, Tests used to evaluate dizziness in primary care, Cmaj 182 (2010), E621-631.

[8] E. Ekvall Hansson, N.O. Mansson and A. Hakansson, Benign paroxysmal positional vertigo among elderly patients in primary health care, Gerontology 51 (2005), 386-389.

[9] C. Evren, N. Demirbilek, M.S. Elbistanli, F. Kokturk and M. Celik, Diagnostic value of repeated Dix-Hallpike and roll maneuvers in benign paroxysmal positional vertigo, $\mathrm{Braz} J$ Otorhinolaryngol 83 (2017), 243-248.

[10] L. Fernandez, H.A. Breinbauer and P.H. Delano, Vertigo and Dizziness in the Elderly, Front Neurol 6 (2015), 144.

[11] J.M. Furman and S.P. Cass, Benign paroxysmal positional vertigo, $N$ Engl J Med 341 (1999), 1590-1596.

[12] K. Hanley, T. O’Dowd and N. Considine, A systematic review of vertigo in primary care, Br J Gen Pract $\mathbf{5 1}$ (2001), 666-671.
[13] E.E. Hansson, N.O. Mansson and A. Hakansson, Balance performance and self-perceived handicap among dizzy patients in primary health care, Scand J Prim Health Care 23 (2005), 215-220.

[14] K. Higashi-Shingai, T. Imai, T. Kitahara, A. Uno, Y. Ohta, A. Horii, S. Nishiike, T. Kawashima, T. Hasegawa and H. Inohara, Diagnosis of the subtype and affected ear of benign paroxysmal positional vertigo using a questionnaire, Acta Otolaryngol 131 (2011), 1264-1269.

[15] M.P. Hilton and D.K. Pinder, The Epley (canalith repositioning) manoeuvre for benign paroxysmal positional vertigo, Cochrane Database Syst Rev 12 (2014), Cd003162.

[16] A. Katsarkas, Dizziness in aging: A retrospective study of 1194 cases, Otolaryngol Head Neck Surg 110 (1994), 296-301.

[17] A.S. Kim, H.J. Fullerton and S.C. Johnston, Risk of vascular events in emergency department patients discharged home with diagnosis of dizziness or vertigo, Ann Emerg Med 57 (2011), 34-41.

[18] L. Kollen, K. Frandin, M. Moller, M. Fagevik Olsen and C. Moller, Benign paroxysmal positional vertigo is a common cause of dizziness and unsteadiness in a large population of 75-year-olds, Aging Clin Exp Res 24 (2012), 317-323.

[19] S.G. Korres, C.E. Papadakis, M.G. Riga, D.G. Balatsouras, D.G. Dikeos and C.R. Soldatos, Sleep position and laterality of benign paroxysmal positional vertigo, $J$ Laryngol Otol 122 (2008), 1295-1298.

[20] K. Mizukoshi, Y. Watanabe, H. Shojaku, J. Okubo and I. Watanabe, Epidemiological studies on benign paroxysmal positional vertigo in Japan, Acta Otolaryngol Suppl 447 (1988), 67-72.

[21] L. Murdin and A.G. Schilder, Epidemiology of balance symptoms and disorders in the community: A systematic review, Otol Neurotol 36 (2015), 387-392.

[22] K. Noda, M. Ikusaka, Y. Ohira, T. Takada and T. Tsukamoto, Predictors for benign paroxysmal positional vertigo with positive Dix-Hallpike test, Int J Gen Med 4 (2011), 809-814.

[23] J.S. Oghalai, S. Manolidis, J.L. Barth, M.G. Stewart and H.A. Jenkins, Unrecognized benign paroxysmal positional vertigo in elderly patients, Otolaryngol Head Neck Surg 122 (2000), 630-634.

[24] P. Perez, V. Franco, P. Cuesta, P. Aldama, M.J. Alvarez and J.C. Mendez, Recurrence of benign paroxysmal positional vertigo, Otol Neurotol 33 (2012), 437-443.

[25] E.G. Piker and G.P. Jacobson, Self-report symptoms differ between younger and older dizzy patients, Otol Neurotol 35 (2014), 873-879.

[26] A. Saxena and M.C. Prabhakar, Performance of DHI score as a predictor of benign paroxysmal positional vertigo in geriatric patients with dizziness/vertigo: A cross-sectional study, PLoS One 8 (2013), e58106.

[27] D.B. Shim, J.H. Kim, K.C. Park, M.H. Song and H.J. Park, Correlation between the head-lying side during sleep and the affected side by benign paroxysmal positional vertigo involving the posterior or horizontal semicircular canal, Laryngoscope 122 (2012), 873-876.

[28] H.J. van der Zaag-Loonen, R.B. van Leeuwen, T.D. Bruintjes and B.C. van Munster, Prevalence of unrecognized benign paroxysmal positional vertigo in older patients, Eur Arch Otorhinolaryngol 272 (2015), 1521-1524.

[29] E. Viirre, I. Purcell and R.W. Baloh, The Dix-Hallpike test and the canalith repositioning maneuver, Laryngoscope 115 (2005), 184-187. 
[30] M. von Brevern, P. Bertholon, T. Brandt, T. Fife, T. Imai, D. Nuti and D. Newman-Toker, Benign paroxysmal positional vertigo: Diagnostic criteria, J Vestib Res 25 (2015), 105-117.

[31] M. von Brevern, A. Radtke, F. Lezius, M. Feldmann, T. Ziese, T. Lempert and H. Neuhauser, Epidemiology of benign paroxysmal positional vertigo: A population based study, J Neurol Neurosurg Psychiatry 78 (2007), 710-715.
[32] M. von Brevern, T. Seelig, H. Neuhauser and T. Lempert, Benign paroxysmal positional vertigo predominantly affects the right labyrinth, J Neurol Neurosurg Psychiatry 75 (2004), 1487-1488.

[33] S. Yetiser and D. Ince, Demographic analysis of benign paroxysmal positional vertigo as a common public health problem, Ann Med Health Sci Res 5 (2015), 50-53. 